\title{
A New Mechanism for Transmissible Prion Diseases
}

\author{
Natallia Makarava, ${ }^{1}$ Gabor G. Kovacs, ${ }^{2}$ Regina Savtchenko, ${ }^{1}$ Irina Alexeeva, ${ }^{3}$ Valeriy G. Ostapchenko, ${ }^{1}$ Herbert Budka, ${ }^{2}$ \\ Robert G. Rohwer, ${ }^{3}$ and Ilia V. Baskakov ${ }^{1,4}$ \\ ${ }^{1}$ Center for Biomedical Engineering and Technology, University of Maryland, Baltimore, Maryland 21201, ${ }^{2}$ Institute of Neurology, Medical University of \\ Vienna, A-1097 Vienna, Austria, ${ }^{3}$ Medical Research Service, Veterans Affairs Medical Center, University of Maryland, Baltimore, Maryland 21201, and \\ ${ }^{4}$ Department of Anatomy and Neurobiology, University of Maryland School of Medicine, Baltimore, Maryland 21201
}

The transmissible agent of prion disease consists of prion protein $(\operatorname{PrP})$ in $\beta$-sheet-rich state $\left(\operatorname{PrP} \mathrm{P}^{\mathrm{Sc}}\right)$ that can replicate its conformation according to a template-assisted mechanism. This mechanism postulates that the folding pattern of a newly recruited polypeptide accurately reproduces that of the $\operatorname{PrP}^{\mathrm{Sc}}$ template. Here, three conformationally distinct amyloid states were prepared in vitro using Syrian hamster recombinant $\operatorname{PrP}(\mathrm{rPrP})$ in the absence of cellular cofactors. Surprisingly, no signs of prion infection were found in Syrian hamsters inoculated with $\mathrm{rPrP}$ fibrils that resembled $\mathrm{PrP}^{\mathrm{Sc}}$, whereas an alternative amyloid state, with a folding pattern different from that of $\mathrm{PrP}^{\mathrm{Sc}}$, induced a pathogenic process that led to transmissible prion disease. An atypical proteinase K-resistant, transmissible PrP form that resembled the structure of the amyloid seeds was observed during a clinically silent stage before authentic PrP $\mathrm{P}^{\mathrm{Sc}} \mathrm{emerged}^{\mathrm{S}} \mathrm{The}$ dynamics between the two forms suggest that atypical proteinase K-resistant $\operatorname{PrP}$ (PrPres) gave rise to $\operatorname{PrP}{ }^{\mathrm{Sc}}$. While no PrP ${ }^{\mathrm{Sc}}$ was found in preparations of fibrils using protein misfolding cyclic amplification with beads (PMCAb), rPrP fibrils gave rise to atypical PrPres in modified PMCAb, suggesting that atypical PrPres was the first product of $\operatorname{PrP}^{\mathrm{C}}$ misfolding triggered by fibrils. The current work demonstrates that a new mechanism responsible for prion diseases different from the $\mathrm{PrP}^{\mathrm{Sc}}$-templated or spontaneous conversion of $\mathrm{PrP} \mathrm{P}^{\mathrm{C}}$ into $\mathrm{PrP}^{\mathrm{Sc}}$ exists. This study provides compelling evidence that noninfectious amyloids with a structure different from that of PrP $\mathrm{P}^{\mathrm{Sc}}$ could lead to transmissible prion disease. This work has numerous implications for understanding the etiology of prion and other neurodegenerative diseases.

\section{Introduction}

Prion diseases, or transmissible spongiform encephalopathies, are fatal neurodegenerative disorders that can arise spontaneously and be inherited or acquired through transmission (Prusiner, 1997). Three general mechanisms have been put forward to explain the diversity in etiology of prion diseases. Spontaneous misfolding and aggregation of the normal cellular isoform of the prion protein $(\operatorname{PrP}), \operatorname{PrP}^{\mathrm{C}}$, into the disease-related infectious isoform, $\operatorname{PrP}^{\mathrm{Sc}}$, is believed to underlie the sporadic forms of prion diseases (Cohen and Prusiner, 1998). Inherited prion diseases have been linked to a number of single point mutations, truncation, or octarepeat expansion mutations in the PRNP gene, with more than 20 disease-inducing mutations identified (Prusiner and Scott, 1997; Kong et al., 2004; Jackson et al., 2009; Sigurdson et al., 2009). In prion diseases acquired through the transmission of the infectious form, $\mathrm{PrP}^{\mathrm{Sc}}$, the abnormal conformation replicates itself in an autocatalytic manner by recruit-

\footnotetext{
Received Dec. 20, 2011; revised March 20, 2012; accepted April 3, 2012.

Author contributions: N.M. and I.V.B. designed research; N.M., G.G.K., R.S., I.A., V.G.0., and R.G.R. performed research; N.M., G.G.K., H.B., and I.V.B. analyzed data; N.M., G.G.K., and I.V.B. wrote the paper.

This work was supported by NIH Grant NSO45585 to I.V.B. and Baltimore Research and Education Foundation. This article is freely available online through the J Neurosci Open Choice option.

Correspondence should be addressed to llia V. Baskakov, Center for Biomedical Engineering and Technology, University of Maryland, 725 West Lombard Street, Baltimore, MD 21201. E-mail: Baskakov@umaryland.edu.

V. G. Ostapchenko's present address J. Allyn Taylor Centre for Cell Biology, Molecular Brain Research Group, Robarts Research Institute, and Department of Physiology and Pharmacology, University of Western Ontario, London, Ontario N6A 5KB, Canada.

DOI:10.1523/JNEUROSCI.6351-11.2012

Copyright $\odot 2012$ the authors $\quad 0270-6474 / 12 / 327345-11 \$ 15.00 / 0$
}

ing and converting the $\operatorname{PrP}^{\mathrm{C}}$ of the host. According to this template-assisted mechanism, the folding pattern of a newly recruited polypeptide chain accurately reproduces that of a $\operatorname{PrP}^{\mathrm{Sc}}$ template (Cohen and Prusiner, 1998).

The current work suggests that a new mechanism responsible for the etiology of transmissible prion diseases exists that is different from those of template-assisted conversion and spontaneous conversion of $\operatorname{PrP}^{\mathrm{C}}$ into $\mathrm{PrP}^{\mathrm{Sc}}$. In the current work, three conformationally distinct and well defined amyloid states were prepared in vitro in the absence of any cellular cofactors using only highly purified full-length Syrian hamster recombinant PrP $(\mathrm{rPrP})$. Surprisingly, no signs of prion infection were found in animals inoculated with $\mathrm{rPrP}$ amyloid state that resembled $\mathrm{PrP}^{\mathrm{Sc}}$, whereas an alternative $\mathrm{rPrP}$ amyloid state, with a folding pattern significantly different from that of $\mathrm{PrP}^{\mathrm{Sc}}$, induced a pathogenic process that eventually led to transmissible prion disease. Accumulation of atypical, proteinase K (PK)-resistant PrP (atypical PrPres) with a PK-resistant core that closely resembled that of the $\mathrm{rPrP}$ fibrils preceded authentic $\operatorname{PrP}^{\mathrm{Sc}}$. While no $\operatorname{PrP}^{\mathrm{Sc}}$ particles were found in preparations of the $\mathrm{rPrP}$ fibrils in serial protein misfolding cyclic amplification with beads (PMCAb), the $\mathrm{rPrP}$ fibrils produced atypical PrPres in modified PMCAb, proving their capability to seed misfolding of $\operatorname{PrP}^{\mathrm{C}}$ and demonstrating their structural difference with authentic $\operatorname{PrP}^{\mathrm{Sc}}$. Moreover, amplification of both brain-derived and fibril-triggered PMCAbderived atypical PrPres was found to be RNA independent, whereas amplification of $\mathrm{PrP}^{\mathrm{Sc}}$ was RNA dependent. Taken together, these results indicate that atypical PrPres was the first 
product of $\mathrm{PrP}^{\mathrm{C}}$ misfolding triggered by fibrils and that the RNAindependent structure of atypical PrPres gave rise to structurally different, RNA-dependent $\mathrm{PrP}^{\mathrm{Sc}}$.

The new mechanism of prion disease introduced here postulates that structures substantially different from that of $\mathrm{PrP}^{\mathrm{Sc}} \mathrm{can}$ induce transmissible disease. The current study raises great concern that structures which are considered noninfectious could lead to a transmissible disease. Because pathological changes associated with diverse neurodegenerative diseases can be induced or transmitted in a prion-like manner through aggregated forms of nonprion proteins (for review, see Aguzzi and Rajendran, 2009; Miller, 2009; Frost and Diamond, 2010), the current work has far-reaching implications.

\section{Materials and Methods}

Ethics statement. This study was carried out in strict accordance with the recommendations in the Guide for the Care and Use of Laboratory Animals of the National Institutes of Health. The protocol was approved by the Institutional Animal Care and Use Committee of the University of Maryland, Baltimore, MD (assurance number A32000-01; permit number: 0309001).

Expression and purification of $r P r P$ and formation of $r P r P$ fibrils. Syrian hamster full-length recombinant $\operatorname{PrP}$ encompassing residues 23-231 was expressed and purified according to a described previously procedure (Bocharova et al., 2005a) with minor modifications (Makarava et al., 2010). Lyophilized rPrP was dissolved in 5 mM HEPES, pH 7.0, immediately before use. To form fibrils for inoculations, the rPrP stock solution was supplemented with $50 \mathrm{~mm}$ MES, pH 6.0, and 0.5 or $2.0 \mathrm{~m}$ guanidine hydrochloride $(\mathrm{GdnHCl})$ and incubated at $37^{\circ} \mathrm{C}$ under continuous agitation. Fibrillation in $0.5 \mathrm{M} \mathrm{GdnHCl}$ was performed with $0.5 \mathrm{mg} / \mathrm{ml} \mathrm{rPrP}$ under $600 \mathrm{rpm}$ horizontal shaking using a DELFIA plate shaker (Wallac), while fibrillation in $2 \mathrm{M} \mathrm{GdnHCl}$ was performed with $0.25 \mathrm{mg} / \mathrm{ml} \mathrm{rPrP}$ under $24 \mathrm{rpm}$ rotation using a Clay Adams Nutator (model 1105). S-fibrils were formed in $2 \mathrm{M} \mathrm{GndCl}$ with $0.5 \mathrm{mg} / \mathrm{ml} \mathrm{rPrP}$ under shaking as described previously (Makarava and Baskakov, 2008). Amyloid formation was confirmed by thioflavin $\mathrm{T}$ fluorescence assay, epifluorescent microscopy, and electron microscopy as described previously (Bocharova et al., 2005a). Fibrils were dialyzed into $10 \mathrm{~mm}$ sodium acetate, $\mathrm{pH}$ 5.0, for analysis or into PBS, pH 7.4, for inoculations. As a control, a rPrP stock solution was diluted in PBS, $\mathrm{pH}$ 7.4, for a final protein concentration of $0.5 \mathrm{mg} / \mathrm{ml}$ and used for inoculation as normally folded $\alpha$-rPrP. Conformation of $\alpha$-rPrP was confirmed by circular dichroism.

Fourier transform infrared spectroscopy. Fourier transform infrared (FTIR) spectroscopy was performed as described previously (Ostapchenko et al., 2010). Briefly, rPrP fibrils were dialyzed in $10 \mathrm{~mm}$ sodium acetate, pH 5.0, loaded into a BioATR II cell of a Bruker Tensor 27 FTIR spectrometer (Bruker Optics), and their infrared spectra were then collected at $2 \mathrm{~cm}^{-1}$ resolution. Five hundred twelve scans were averaged for each fibril species and treated with Opus software (Bruker Optics) to obtain FTIR second derivative spectra.

Bioassay. For the first passage, weanling Golden Syrian hamsters (all males) were inoculated intracerebrally with three different $\mathrm{rPrP}$ amyloid states or $\alpha$-rPrP described above (see Expression and purification of $\mathrm{rPrP}$ and formation of $\mathrm{rPrP}$ fibrils). Each animal was anesthetized with $2 \%$ $\mathrm{O}_{2} / 4$ minimum alveolar concentration (MAC) isoflurane before receiving $50 \mu \mathrm{l}$ of inoculum. Starting from the third month postinoculation, hamsters were observed daily for disease. They were killed at $664-723 \mathrm{~d}$ postinoculation without any signs of clinical disease and their brains were removed aseptically and saved for analysis and second passage. For the second passage, $10 \%$ brain homogenates $(\mathrm{BHs})$ prepared by sonication in PBS, pH 7.4 (Makarava et al., 2010), were dispersed by an additional $30 \mathrm{~s}$ of sonication immediately before inoculation. Each hamster received $50 \mu \mathrm{l}$ of $10 \% \mathrm{BH}$ inoculum intracerebrally under $2 \% \mathrm{O}_{2} / 4 \mathrm{MAC}$ isoflurane anesthesia. After inoculation, hamsters were observed daily for disease using a 'blind' scoring protocol.

Proteinase K digestion. Brains were collected aseptically and cut in half with disposable scalpels. One half was used to prepare $10 \%$ BHs in PBS or conversion buffer as described previously (Makarava et al., 2010), while the second half was stored at $-80^{\circ} \mathrm{C}$ for future analysis or fixed in formalin for histopathology. For the PK digestion of BH in sarcosyl, an aliquot of $10 \% \mathrm{BH}$ was mixed with an equal volume of $4 \%$ sarcosyl in PBS supplemented with $50 \mathrm{~mm}$ Tris, $\mathrm{pH}$ 7.5, and digested with $20 \mu \mathrm{g} / \mathrm{ml} \mathrm{PK}$ for $30 \mathrm{~min}$ at $37^{\circ} \mathrm{C}$ with $1000 \mathrm{rpm}$ shaking using a DELFIA plate shaker (Wallac) placed in $37^{\circ} \mathrm{C}$ incubator. PK digestion was stopped by adding SDS sample buffer and heating the samples for $10 \mathrm{~min}$ in a boiling water bath. For PK digestion of recombinant fibrils, fibrils were diluted to 10 $\mu \mathrm{g} / \mathrm{ml}$ in $1 \%$ normal $\mathrm{BH}(\mathrm{NBH})$ in conversion buffer supplemented with $0.25 \%$ SDS and digested with 10 or $50 \mu \mathrm{g} / \mathrm{ml} \mathrm{PK}$ for $1 \mathrm{~h}$ at $37^{\circ} \mathrm{C}$. After loading onto NuPAGE 12\% Bis-Tris gels and transfer to PVDF membrane, PrP was detected with 3F4 (epitope 109-112), D18 (epitope 133157), or SAF-84 (epitope 160-170) antibody, as indicated. Density profiles for Western blots were generated using WCIF ImageJ software (National Institutes of Health).

Protein misfolding cyclic amplification with beads. Ten percent NBH from healthy hamsters was prepared as described previously (Makarava et al., 2011) and used as a substrate for PMCA with beads (GonzalezMontalban et al., 2011a). For the first round, $10 \mu \mathrm{l}$ of $10 \% \mathrm{BH}$ from inoculated animals was added to $90 \mu \mathrm{l}$ of NBH. The standard sonication program consisted of $30 \mathrm{~s}$ sonication pulses delivered at 50\% power efficiency applied every $30 \mathrm{~min}$ during a $24 \mathrm{~h}$ period. For each subsequent round, $10 \mu \mathrm{l}$ of the reaction from the previous round were added to $90 \mu \mathrm{l}$ of fresh substrate. Each PMCAb reaction was carried out in the presence of three 3/32" Teflon beads (McMaster Carr). To analyze production of PK-resistant PrP material in PMCAb, $10 \mu \mathrm{l}$ of sample were supplemented with $5 \mu \mathrm{l}$ of SDS and $5 \mu \mathrm{l}$ of PK to a final concentration of SDS and PK of $0.25 \%$ and $50 \mu \mathrm{g} / \mathrm{ml}$ respectively, followed by incubation at $37^{\circ} \mathrm{C}$ for $1 \mathrm{~h}$. The digestion was terminated by addition of SDS-sample buffer and heating the samples for $10 \mathrm{~min}$ in a boiling water bath. Samples were loaded onto NuPAGE 12\% Bis-Tris gels, transferred to PVDF membrane, and probed with 3F4 or SAF-84 antibodies.

Protein misfolding cyclic amplification with partially deglycosylated substrate. To produce substrate for protein misfolding cyclic amplification with partially deglycosylated substrate (dgPMCAb), 10\% NBH from healthy hamsters prepared for PMCAb (see Protein misfolding cyclic amplification with beads) was treated with peptide $N$-glycosidase F (PNGase F) (New England BioLabs, glycerol-free) as follows. After preclearance of NBH at $500 \times g$ for $2 \mathrm{~min}, 1500 \mathrm{U} / \mathrm{ml}$ PNGase F was added to the supernatant, and the reaction was incubated on a rotator at $37^{\circ} \mathrm{C}$ for $5 \mathrm{~h}$. The resulting substrate was used in dgPMCAb using sonication conditions as described for PMCAb. To prepare RNA-depleted normal brain homogenate, $\mathrm{NBH}$ was precleared at $500 \times g$ for $2 \mathrm{~min}$, and then 100 $\mu / \mathrm{ml}$ RNase A (Sigma, catalog no. R4875) was added to the supernatant and the reaction was incubated on a rotator at $37^{\circ} \mathrm{C}$ for $1 \mathrm{~h}$ as described previously (Gonzalez-Montalban et al., 2011b). Lack of RNA in RNAdepleted NBH was confirmed by RNA analysis in agarose gel.

Histopathological studies. Formalin-fixed brain halves divided at the midline (right hemisphere), spinal cord, and spleen were processed for hematoxylin-eosin (H\&E) stain as well as for immunohistochemistry for PrP, using the mouse monoclonal anti-PrP antibody 3F4 (1:1000, Covance) and anti-glial fibrillar acidic protein (GFAP; 1:3000, Dako). Blocks were treated in formic acid ( $96 \%$ ) before being embedded in paraffin. For detection of disease-associated $\mathrm{PrP}$, we applied a pretreatment of $30 \mathrm{~min}$ hydrated autoclaving at $121^{\circ} \mathrm{C}$ followed by $5 \mathrm{~min}$ in $96 \%$ formic acid. We evaluated all tissues for the presence of inflammation and PrP immunoreactivity, and the brain for the presence of spongiform change and degree of gliosis. Degree of spongiform change, neuronal loss, and gliosis and intensity of PrP immunostaining were semiquantitatively evaluated $(0$, none; 1 , mild; 2 , moderate; 3 , severe) in the following anatomical regions as described previously: frontal cortex, hippocampus, caudateputamen, thalamus, brainstem, and cerebellum (Makarava et al., 2010). Lesion profiles were obtained by averaging scores of spongiform change, neuronal loss, and gliosis for each anatomical region and animal group.

Conformational stability assay. Ten percent BHs were sonicated for $30 \mathrm{~s}$ at $50 \%$ efficiency within Misonix-4000 microplate horn filled with 350 $\mathrm{ml}$ of water. Then the samples were diluted 2 -fold with conversion buffer and incubated with various concentrations of $\mathrm{GdnHCl}$ in $\mathrm{PBS}, \mathrm{pH}$ 7.4, 


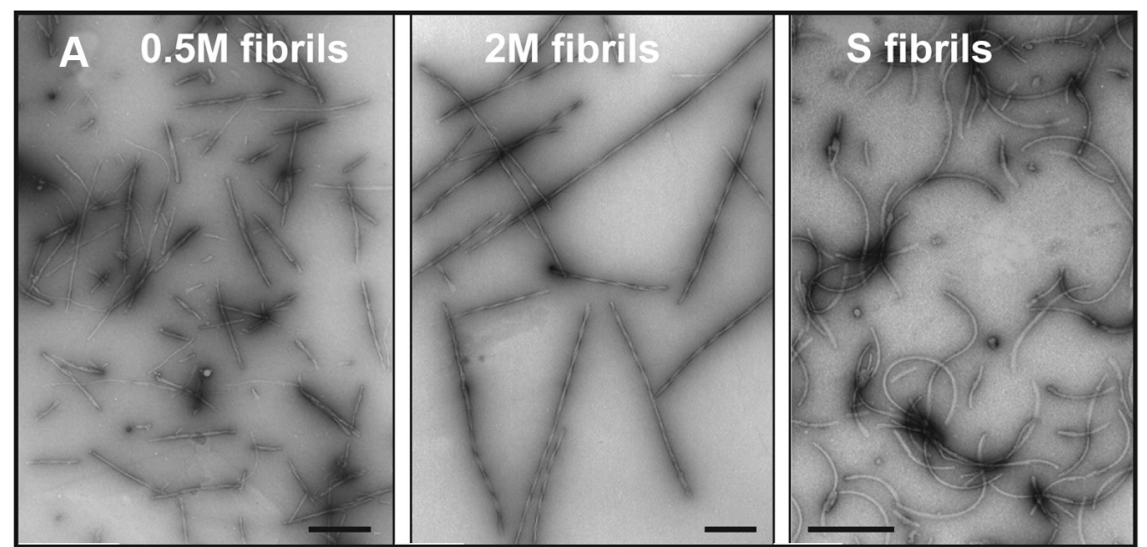

B $\mathrm{GdnHCl}, \mathrm{M}$

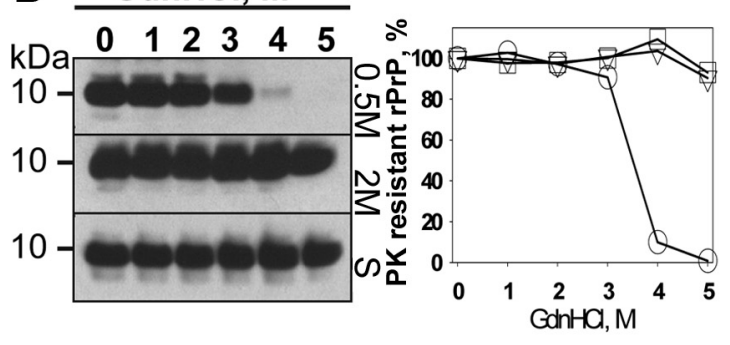

C

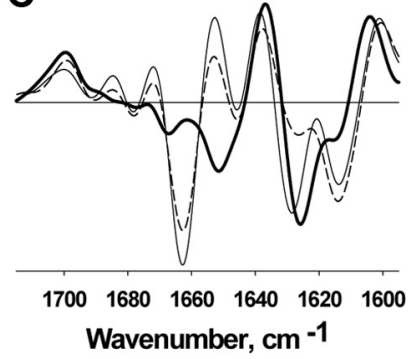

Figure 1. Analysis of $\mathrm{rPrP}$ fibrils produced in vitro. $\boldsymbol{A}$, Negative staining electron microscopy of $0.5 \mathrm{~m}$ fibrils (left), $2 \mathrm{~m}$ fibrils (middle), or $S$ fibrils (right). Scale bars, $0.1 \mu \mathrm{m}$. B, Analysis of fibril conformational stability. Western blots (left) and the conformational stability profiles (right) for fibrils subjected to $\mathrm{GdnHCl}$-induced denaturation and treated with PK: $0.5 \mathrm{~m}$ fibrils (circles), 2 M fibrils (triangles), S fibrils (squares). C, Analysis of fibril secondary structure using FTIR spectroscopy. Second derivatives of FTIR spectra for $0.5 \mathrm{~m}$ fibrils (thin line), $2 \mathrm{~m}$ fibrils (dashed line), or $\mathrm{S}$ fibrils (bold line).

for $1 \mathrm{~h}$ at room temperature. After incubation, all samples were diluted with nine volumes of $2 \%$ sarcosyl in PBS and incubated for additional $1 \mathrm{~h}$ at room temperature. Then, $20 \mu \mathrm{g} / \mathrm{ml}$ PK were added to each sample followed by incubation for $1 \mathrm{~h}$ at $37^{\circ} \mathrm{C}$ with $1000 \mathrm{rpm}$ shaking using a DELFIA plate shaker (Wallac). The PK digestion was stopped by $2 \mathrm{~mm}$ PMSF, and the samples were precipitated by addition of 4 volumes of ice-cold acetone and overnight incubation at $-20^{\circ} \mathrm{C}$. The following day, samples were centrifuged for $30 \mathrm{~min}$ at 13,000 rpm in an AccuSpin Micro centrifuge (Fisher Scientific), the supernatants were aspirated, and the pellets were dried for $30 \mathrm{~min}$ at room temperature, dissolved in $1 \times$ SDS-loading buffer, denatured by incubation in boiling water bath for 10 min, and loaded onto NuPAGE $12 \%$ Bis-Tris gels. After transfer to PVDF, PrP was detected with 3F4 antibody.

To analyze the conformational stability of $\mathrm{rPrP}$ fibrils, dialyzed preparations of fibrils were supplemented with various concentrations of $\mathrm{GdnHCl}$ in $50 \mathrm{~mm} \mathrm{MES}, \mathrm{pH} 6.0$, incubated for $1 \mathrm{~h}$ at room temperature, and then diluted out of $\mathrm{GdnHCl}$ with $1 \% \mathrm{NBH}$ in conversion buffer before PK digestion with $2 \mu \mathrm{g} / \mathrm{ml}$ PK for $1 \mathrm{~h}$ at $37^{\circ} \mathrm{C}$. The reaction was stopped by PMSF, precipitated by acetone, and analyzed by Western blot with SAF-84 antibody. Signal intensity of Western blots was measured using WCIF ImageJ software (National Institute of Health).

Deglycosylation of PrPres. Removal of N-linked glycans was performed as described previously (Deleault et al., 2008) using glycerol-free PNGase F and supplied buffers (New England BioLabs). Ten percent BH or dgPMCAb product were mixed with equal volume of $4 \%$ sarcosyl in PBS, pH 7.4, digested with $20 \mu \mathrm{g} / \mathrm{ml}$ or $50 \mu \mathrm{g} / \mathrm{ml} \mathrm{PK}$ as described above, deglycosylated following the procedure described previously (Makarava et al., 2010), and assayed by Western blot with SAF-84 or D18 antibody.

\section{Results}

Generating three conformationally distinct amyloid states Three experimental protocols that produce three well defined and conformationally distinct amyloid states were employed as described previously (Makarava and Baskakov, 2008; Sun et al.,
2008; Ostapchenko et al., 2010). Briefly, $\mathrm{rPrP}$ fibrils were formed in $0.5 \mathrm{M} \mathrm{GdnHCl}$ (referred to as $0.5 \mathrm{M}$ fibrils) in $2 \mathrm{M} \mathrm{GdnHCl}$ under rotation (referred to as $2 \mathrm{M}$ fibrils), or in $2 \mathrm{M} \mathrm{GdnHCl}$ under shaking (referred to as S-fibrils, Fig. 1A). rPrP expression, purification, and fibrillation procedures were conducted in a laboratory that was never exposed to transmissible spongiform encephalopathies (TSEs). In agreement with the previous studies, $0.5 \mathrm{M}$ fibrils were substantially shorter in length than the 2 m fibrils (Sun et al., 2008), whereas $\mathrm{S}$ fibrils displayed a characteristic curvy shape (Fig. 1A) (Makarava and Baskakov, 2008; Ostapchenko et al., 2010). The differences in length between $0.5 \mathrm{M}$ and $2 \mathrm{M}$ fibrils were presumably due to higher intrinsic fragility of the $0.5 \mathrm{M}$ fibrils (Sun et al., 2008). As judged from the $\mathrm{GdnHCl}$-induced denaturation assay, $2 \mathrm{M}$ or $\mathrm{S}$ fibrils were substantially more stable than the fibrils produced in $0.5 \mathrm{M}$ $\mathrm{GdnHCl}$ (Fig. $1 B$ ). In agreement with previous studies that employed FTIR ( $\mathrm{Ma}$ karava and Baskakov, 2008; Ostapchenko et al., 2010), $0.5 \mathrm{M}$ and $2 \mathrm{M}$ but not $\mathrm{S}$ fibrils were characterized by a large peak at 1663 $\mathrm{cm}^{-1}$, which is typically assigned to $\beta$ - or $\gamma$-turns (Fig. $1 C$ ). In S fibrils, the cross- $\beta$ structure was represented by a peak at $1625 \mathrm{~cm}^{-1}$ with a shoulder at $1614 \mathrm{~cm}^{-1}$, whereas in $0.5 \mathrm{M}$ or $2 \mathrm{M}$ fibrils, the cross- $\beta$ structure exhibited two peaks at $1628 \mathrm{~cm}^{-1}$ and $1614 \mathrm{~cm}^{-1}$, where the latter is believed to represent more rigid $\beta$-sheets than the former. As judged from electron microscopy, the interface surface between filaments was greater in $2 \mathrm{M}$ than in $0.5 \mathrm{M}$ or S fibrils (Fig. $1 A$ ), consistent with the larger fraction of rigid $\beta$-structure in $2 \mathrm{~m}$ fibrils as evident from a predominant peak at $1614 \mathrm{~cm}^{-1}$ (Fig. 1C).

Fibrils $(0.5 \mathrm{M})$ seeded formation of atypical PrPres in animals The three $\mathrm{rPrP}$ fibrillar types or $\mathrm{rPrP}$ folded into normal $\alpha$-helical conformation $(\alpha$-rPrP) were inoculated into Syrian hamsters. No clinical signs were observed in any animal groups until old age (664-723 d postinoculation), when all animals were killed. However, six of seven animals inoculated with $0.5 \mathrm{M}$ fibrils showed atypical, PK-resistant, C-terminal bands of $\sim 13,16$, and $23 \mathrm{kDa}$ (designated as atypical PrPres) (Fig. 2B). Atypical PrPres was immunoreactive with SAF-84 antibody (epitope 160-170), but not with 3F4 antibody (epitope 109-112) (Fig. 2 A,B). Upon treatment with PNGase F, the three PK-resistant bands of 23, 16, and $13 \mathrm{kDa}$ in atypical PrPres merged into a single band of $\sim 13$ $\mathrm{kDa}$, illustrating that these three bands represent diglycosylated, monoglycosylated, and unglycosylated forms of a single PKresistant fragment, respectively (Fig. 2D). This fragment was immunoreactive with SAF-84 (epitope 160-170) antibody, but not with D18 (epitope 133-157) antibody (Fig. 2D). The PK-resistant core of $0.5 \mathrm{M} \mathrm{rPrP}$ fibrils consisted of two major fragments of 12 and $10 \mathrm{kDa}$ (Fig. 2D). As judged from mass spectroscopy and epitope mapping, the 12 and $10 \mathrm{kDa}$ fragments were previously found to encompass residues $\sim 138-231$ and $\sim 152-231$, respectively (Bocharova et al., 2005b). Consistent with these studies, both PK-resistant fragments were immunoreactive with SAF-84 
antibody, but only the $12 \mathrm{kDa}$ fragment reacted with D18 antibody (Fig. 2D). In summary, as judged from the epitope mapping, the PK-resistant core of the atypical PrPres matched the $10 \mathrm{kDa}$ band of $\mathrm{rPrP}$ fibrils and presumably encompassed residues $\sim 152-231$. The difference between PAGE-mobility of the atypical PrPres and the $10 \mathrm{kDa}$ rPrP fragment was likely due to a glycosylphosphatidylinositol (GPI) anchor in the first one.

In addition to atypical PrPres, four of six animals inoculated with $0.5 \mathrm{M}$ fibrils showed variable amounts of PK-resistant material with a band-shift typical for authentic $\operatorname{PrP}^{\mathrm{Sc}}$ that could be detected by 3F4 and the C-terminal antibodies (Fig. 2A). After three serial PMCAb (sPMCAb) rounds, brain homogenates from all six animals that were positive for atypical PrPres showed $\mathrm{PrP}^{\mathrm{Sc}}$ immunoreactive with $3 \mathrm{~F} 4$ (Fig. 2C). In contrast, $\mathrm{BH}$ from one animal that lacked atypical PrPres did not show any detectible $\mathrm{PrP}^{\mathrm{Sc}}$ even after three sPMCAb rounds.

No animals from the groups inoculated with $2 \mathrm{M}$ fibrils, S-fibrils, or $\alpha$-rPrP showed atypical PrPres or $\operatorname{PrP}^{\mathrm{Sc}}$ in their brain material (Fig. 2A,B). Furthermore, no PK-resistant materials were detected in any animals from these three groups after three PMCAb rounds (Fig. 2C). In summary, we concluded that six of seven animals were infected upon inoculation of 0.5 $\mathrm{M}$ fibrils, whereas no signs of prion infection could be detected upon inoculation of $2 \mathrm{M}$ fibrils, S-fibrils, or $\alpha$-rPrP.

\section{Transition from atypical PrPres to $\mathrm{PrP}^{\mathrm{Sc}}$}

To test whether the pathogenic process triggered by $0.5 \mathrm{M}$ fibrils leads to transmissible prion disease, brain material from animals $\# 3$, \#4, \#5, and \#7 was selected for the second passage. BHs from these animals contained large (animal \#3), medium (\#7), small (\#5), or undetectable (\#4) amounts of $\operatorname{PrP} \mathrm{P}^{\mathrm{Sc}}$, but approximately equal amounts of atypical PrPres. Animals from all four groups developed clinical signs of prion disease (Fig. 2 E). All four groups showed similar clinical symptoms, including startle response, difficulty in righting themselves, substantially reduced activity, and obesity. After the first signs, the clinical disease progressed very slowly, which resembled the slow progression of disease reported for two other synthetic strains, SSLOW and LOTSS (Makarava et al., 2010, 2011).

The incubation time to clinical disease correlated well with the amounts of $\operatorname{PrP}^{\mathrm{Sc}}$ in the inoculums (Fig. $2 A, E$ ). The shortest incubation time $(347 \pm 7 \mathrm{~d})$ was found for the animal group inoculated with $\mathrm{BH} \# 3$, whereas the longest incubation time $(512 \pm 82 \mathrm{~d})$ and incomplete attack rate ( 5 out 7 animals showed clinical signs) were observed for the group inoculated with $\mathrm{BH} \# 4$ (Fig. 2A,E). Because the amounts of atypical PrPres in four BHs were approximately the same, these results suggest that the differences in the incubation time and the attack rate were likely attributed to the differences in the amounts of $\operatorname{PrP}^{\mathrm{Sc}}$.

The brain materials from four animal groups were analyzed by Western blot using SAF-84 antibody that detects both atypical
PrPres and $\mathrm{PrP}^{\mathrm{Sc}}$ (Fig. 3). Because of the overlaps between the diglycosylated and monoglycosylated atypical PrPres with the monoglycosylated and unglycosylated $\mathrm{PrP}^{\mathrm{Sc}}$, respectively (Fig. $3 C$, presented as a schematic diagram), the PK-resistant profiles of atypical PrPres and $\mathrm{PrP}^{\mathrm{Sc}}$ mixtures consisted of four bands (Fig. $3 A, B$ ). Nevertheless, the relative amounts of atypical PrPres versus $\mathrm{PrP}^{\mathrm{Sc}}$ could be estimated based on the peak ratios (Fig. $3 B$ ). After careful analysis of the individual PK resistant profiles, several important observations can be made.

First, substantial amounts of atypical PrPres observed in animals from the first and second passages for up to $665 \mathrm{~d}$ postinoculation indicate that this form is self-replicating and its replication does not require the assistance of $\operatorname{PrP}^{\mathrm{Sc}}$ (Figs. $2 A, B$, $3 A$ ). Second, the observations that (1) atypical PrPres appeared before $\mathrm{PrP} \mathrm{P}^{\mathrm{Sc}}$, (2) $\mathrm{PrP}^{\mathrm{Sc}}$ was never detected in animals negative for atypical PrPres, and (3) brain material from animals positive for atypical PrPres always gave rise to $\mathrm{PrP}^{\mathrm{Sc}}$ during serial transmission suggested that atypical PrPres was a precursor of $\operatorname{PrP}^{\mathrm{Sc}}$ (Fig. $2 A-C$ ). Third, the group inoculated with $\mathrm{BH} \# 3$ (with the largest amounts of $\mathrm{PrP}^{\mathrm{Sc}}$ ) showed the highest $\mathrm{PrP}^{\mathrm{Sc}}$ /atypical PrPres ratio when compared to the other three groups. The group inoculated with $\mathrm{BH} \# 4$ (with undetectable amounts of $\mathrm{PrP}^{\mathrm{Sc}}$ ) showed the lowest PrP $\mathrm{Sc}^{\mathrm{S}}$ atypical PrPres ratio (Fig. $3 A, B$ ). Furthermore, the $\mathrm{PrP}^{\mathrm{Sc}}$ /atypical PrPres ratio was variable not only between the four groups but also within individual groups (Fig. $3 A$ ). The greatest variation was observed for the group inoculated with $\mathrm{BH} \# 4$. Significant variations in $\mathrm{PrP}^{\mathrm{Sc}}$ /atypical PrPres ratios at the end of the first and second passages suggest that the seeding of $\operatorname{PrP}^{\mathrm{Sc}}$ by 
A
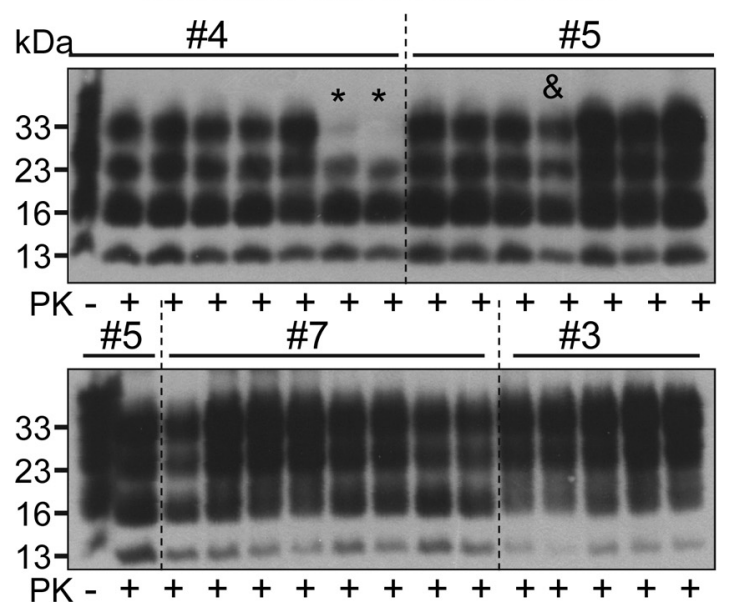

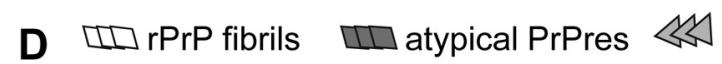

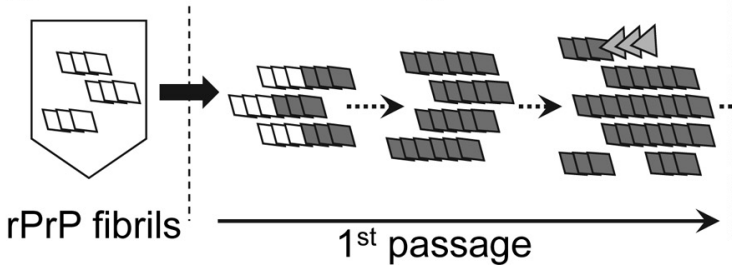

Figure 3. Analysis of brain materials from the second passage. $\boldsymbol{A}$, Western blots of brain material from the animals of second passage inoculated with $\mathrm{BHs} \# 3, \# 4, \# 5$, and \#7 and stained with SAF-84. Two animals marked by asterisks in the group \#4 were asymptomatic up to $665 \mathrm{~d}$ postinoculation. The animal marked with ampersand (\&) was euthanized at $534 \mathrm{~d}$ postinoculation due to an unrelated health problem. $B$, PK resistance profiles for individual animals from two groups: those inoculated with BH \#4 (top panel) or BH \#3 (bottom panel). Four peaks at 33, 23, 16, and $13 \mathrm{kDa}$ represent six PK-resistant bands. C, Schematic representation of the PK resistance profile showing overlap between atypical PrPres and PrP ${ }^{\mathrm{Sc}}$, where atypical PrPres and PrP ${ }^{\mathrm{Sc}}$ are represented by gray and black boxes, respectively. D, Hypothetical mechanism illustrating genesis of $\mathrm{PrP}{ }^{\mathrm{Sc}}$ in animals inoculated with $\mathrm{rPrP}$ fibrils. During the first passage, rPrP fibrils seeded atypical PrPres, a new transmissible form of PrP capable of self-replicating without detectible clinical signs. Replication of atypical PrPres occasionally produces $\mathrm{PrP}{ }^{\mathrm{Sc}}$ in seeding events that appeared to be relatively rare and stochastic, and are described by a deformed templating mechanism (Makarava et al., 2009, 2011). PrP ${ }^{\text {Sc }}$ replicates faster than atypical PrPres and eventually replaces its ancestor.

atypical PrPres appeared to be a stochastic event. Fourth, $\operatorname{PrP}{ }^{S c}$ was predominantly diglycosylated, whereas atypical PrPres was predominantly monoglycosylated (Figs. $2 A, D$ ). This observation illustrates that these two forms had different preferences in recruiting the three $\operatorname{PrP}^{\mathrm{C}}$ glycoforms and supports the notion that the two forms were structurally different. Fifth, accumulation of atypical PrPres alone was not sufficient for development of clinical disease. In support of this notion, two animals from the group inoculated with $\mathrm{BH} \# 4$ that showed large amounts of atypical PrPres but very little if any $\mathrm{PrP}^{\mathrm{Sc}}$ were asymptomatic (Fig. $3 A$ ). Sixth, because atypical PrPres was eventually replaced by $\mathrm{PrP}^{\mathrm{Sc}}$ (Fig. 3A), $\mathrm{PrP}^{\mathrm{Sc}}$ appears to replicate faster and outcompete the atypical PrPres.

\section{Serial transmission of $0.5 \mathrm{M}$ fibrils leads to a new disease phenotype}

Histopathological studies of animals from the second passage revealed characteristic signs of TSE infection, including spongiform degeneration, neuronal loss, reactive astrogliosis, and deposition of disease-associated PrP in the brains without signs of inflammatory infiltration. Spongiform change, neuronal loss, and reactive astrogliosis were predominantly found in the thalamus and the brainstem followed by caudate-putamen (Fig. 4 $A, B)$. However, they were noted also in the frontal cortex, hip- pocampus, and, to a lesser extent, in the cerebellum. A diffuse/synaptic type of immunoreactivity was observed in all examined subregions (Fig. 4B). In addition, perineuronal deposits were found predominantly in the thalamus, basal ganglia, and deeper layers of the cortex (Fig. 4C). Prominent large plaques and amorphous deposits were present in the subpial, periventricular, and periaqueductal subependymal regions (Fig. 4C). Furthermore, small PrP plaques were observed around vessels, mainly in the white matter of the cerebellum (Fig. 4C). The new synthetic strain that emerged upon inoculation of $0.5 \mathrm{~m}$ fibrils will be designated as S05.

Biochemical properties of $\mathrm{S} 05 \mathrm{PrP}^{\mathrm{Sc}}$ were assessed using several assays. While S05 $\mathrm{PrP}^{\mathrm{Sc}}$ and $263 \mathrm{~K} \mathrm{PrP}^{\mathrm{Sc}}$ showed very similar PK resistance (Fig. 5A), S05 PrP ${ }^{\mathrm{Sc}}$ was conformationally more stable than 263K PrP ${ }^{\text {Sc }}$ (Fig. 5B). PNGase treatment revealed that the size of the PK-resistant core of $\mathrm{S} 05 \mathrm{PrP}^{\mathrm{Sc}}$ was slightly shorter than that of $263 \mathrm{~K} \mathrm{PrP}^{\mathrm{Sc}}$ (Fig. 5C). Overall, taking aside strain-specific differences, biochemical assays confirmed that $\mathrm{S} 05 \mathrm{PrP}^{\mathrm{Sc}}$ exhibits physical features typical for authentic $\operatorname{PrP}^{\mathrm{Sc}}$.

\section{Preparation of $0.5 \mathrm{M}$ fibrils lacks any detectible PrP $\mathrm{P}^{\mathrm{Sc}}$}

An alternative to the hypothesis that noninfectious $\mathrm{rPrP}$ fibrils gave rise to $\mathrm{PrP}^{\mathrm{Sc}}$ is the hypothesis that the preparation of 0.5 $\mathrm{M}$ fibrils contains small amounts of $\operatorname{PrP} \mathrm{P}^{\mathrm{Sc}}$. To test whether small amounts of $\operatorname{PrP}{ }^{\mathrm{Sc}}$ were present in the preparations of $0.5 \mathrm{M}$ fibrils, we employed sPMCAb protocol capable of detecting single $\mathrm{PrP}^{\mathrm{Sc}}$ particles. First, we demonstrated that miniscule amounts of $\mathrm{S} 05 \mathrm{PrP}^{\mathrm{Sc}}$ can be efficiently detected by sPMCAb. Ten percent $\mathrm{BHs}$ prepared from clinically ill animals from the second passage of S05 were diluted in 10-fold serial steps, and then aliquots from each dilution were used to seed sPMCAb reactions. In $100 \mu \mathrm{l}$ of PMCAb reaction volume, $10^{10}$ - and $10^{11}$-fold diluted S05 brain material was detected with $100 \%$ and $66 \%$ success rate, respectively (Fig. $6 \mathrm{~A}$ ). The reactions seeded with $10^{12}$-fold or higher dilutions were all negative. Five sPMCAb rounds were sufficient for amplification of the highest dilutions of S05 brain material to the detectible level. An increase in the number of sPMCAb rounds did not improve the success rate in the reactions seeded with highly diluted brain material (Fig. 6A). These results illustrate that the limiting dilution was reached at $10^{11}$-fold dilution. At $10^{11}$-fold dilution there is a $66 \%$ chance of finding at least one PMCAb-active $\mathrm{PrP}^{\mathrm{Sc}}$ particle per $100 \mu \mathrm{l}$ of reaction volume. This experiment demonstrated that $\mathrm{PPMCAb}$ can effectively detect as little as a single $\mathrm{S} 05 \mathrm{PrP}^{\mathrm{Sc}}$ particle.

In the next series of experiments, sPMCAb was employed to detect $\operatorname{PrP}^{\mathrm{Sc}}$ in the preparations of $0.5 \mathrm{M} \mathrm{rPrP}$ fibrils. Multiple sPMCAb reactions were seeded with $0.5 \mathrm{M}$ fibrils from two independent amyloid preparations. No positive signals were detected in any reaction after six sPMCAb rounds (Fig. 6B). As judged 
A

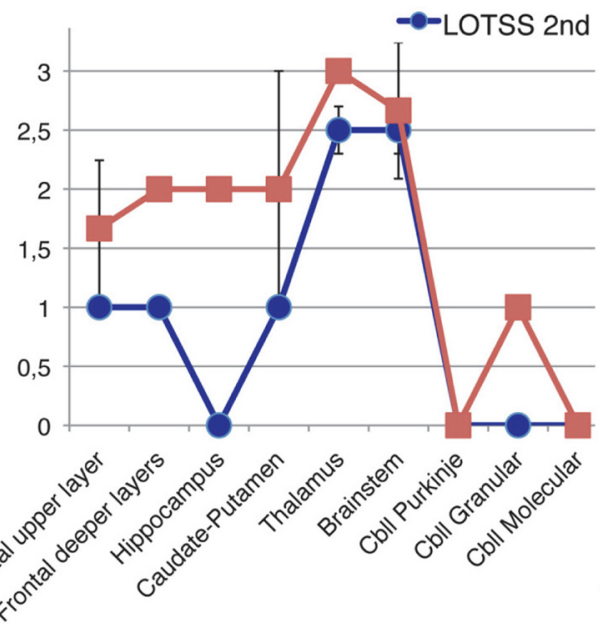

B
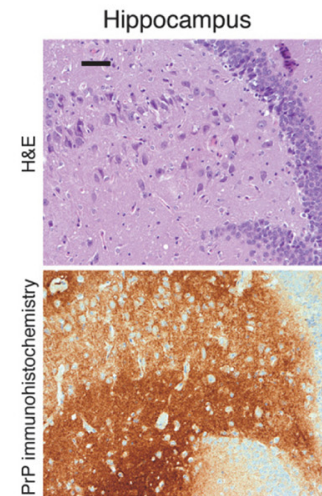

C

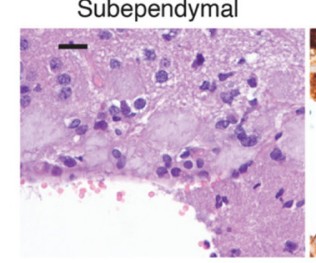

H\&E/Plaques
Frontal

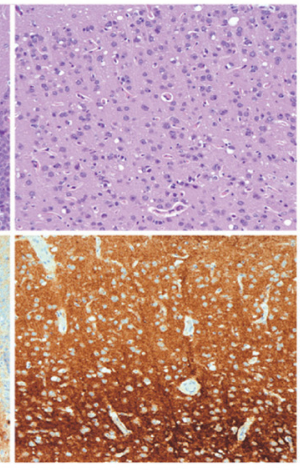

Subependymal

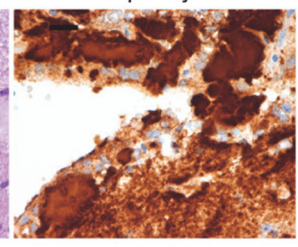

PrP IHC/Plaques

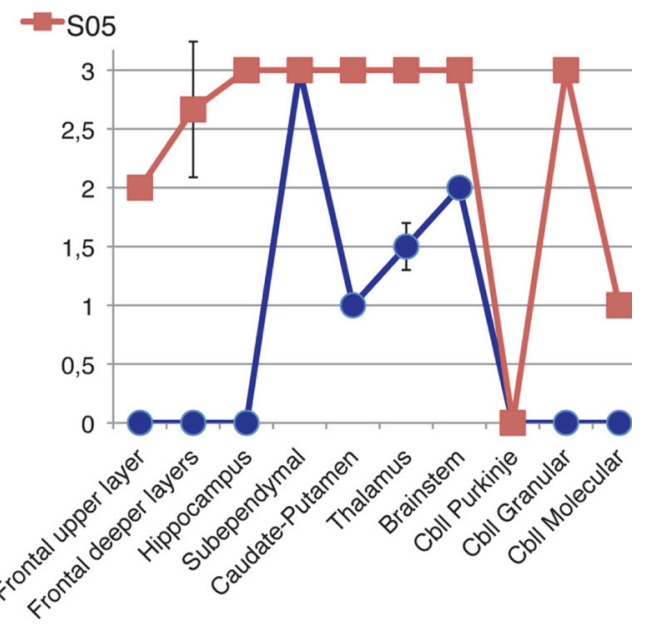

Thalamus

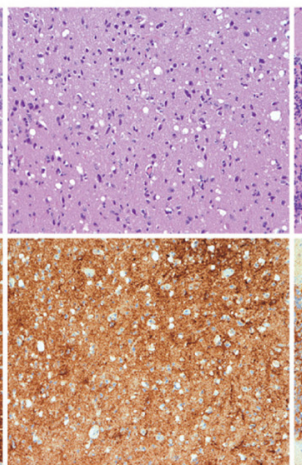

Cerebellum

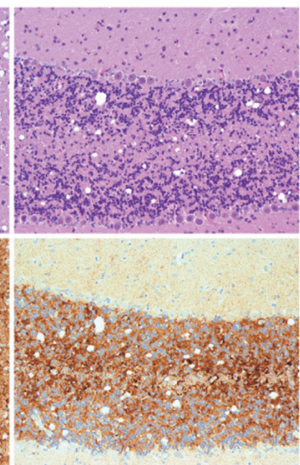

Cerebellum white matter

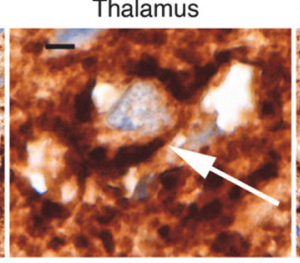

PrP IHC/Perineuronal

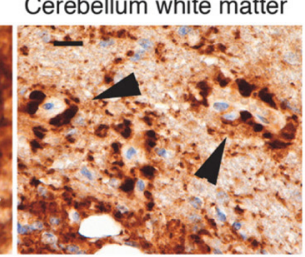

PrP IHC/Perivascular

Figure 4. Histopathological analysis. $A$, Lesion profile (left) and PrP immunopositivity score (right) in hamsters inoculated with BH \#3 (squares, red lines). The histopathological profiles for the second passage of LOTSS-inoculated hamsters (circles, blue lines) are provided as a reference (Makarava et al., 2011). B, Comparison of spongiform changes in the hippocampus, frontal cortex, thalamus, and cerebellum stained with H\&E (top) or anti-PrP 3F4 antibody (bottom) (scale bar, $100 \mu \mathrm{m}$ for all panels). C, Plaques in the brain subependymal region stained with H\&E and 3F4 antibody (scale bar, $30 \mu \mathrm{m}$ ), perineuronal PrP immunoreactivity (indicated by a white arrow) in the thalamus (scale bar, $10 \mu \mathrm{m}$ ), and perivascular PrP plaques (indicated by black arrowheads) in the cerebellar white matter (scale bar, $10 \mu \mathrm{m}$ ).

from the previous experiment, five sPMCAb rounds were sufficient to detect $\mathrm{S} 05 \mathrm{PrP}^{\mathrm{Sc}}$ at the level of a single particle in $100 \mu$ l of reaction volume. To rule out the possibility that the negative results in detecting $\mathrm{PrP}^{\mathrm{Sc}}$ in the preparations of $0.5 \mathrm{M}$ fibrils were due to an inhibitory effect of fibrils on $\mathrm{PrP}^{\mathrm{Sc}}$ amplification, $\mathrm{sPMCAb}$ reactions were seeded with $0.5 \mathrm{M}$ fibrils mixed with $10^{10}$-fold diluted S05 brain material. This dilution was the highest at which 100\% sPMCAb reactions were positive. Three independent sPMCAb reactions were conducted, and all were positive (Fig. 6B). This experiment confirmed that the presence of $\mathrm{rPrP}$ fibrils did not diminish the sensitivity of detection of $\mathrm{S} 05 \mathrm{PrP}^{\mathrm{Sc}}$ by sPMCAb. These experiments revealed that the preparations of $\mathrm{rPrP}$ amyloid fibrils did not contain any $\mathrm{PrP}^{\mathrm{Sc}}$ particles that could be detected by sPMCAb. In our experience and consistent with the previously published data (Saa et al., 2006; Makarava et al., 2012), sPMCAb is up to $\sim 4000$-fold more sensitive than bioassay. These experiments also showed that $0.5 \mathrm{M}$ fibrils failed to convert $\operatorname{PrP}^{\mathrm{C}}$ into $\operatorname{PrP}^{\mathrm{Sc}}$ in $\mathrm{SPMCAb}$ if used as a source of seeds.

\section{Atypical PrPres could be amplified in modified PMCAb using partially deglycosylated $\operatorname{PrP}^{\mathrm{C}}$}

While atypical PrPres appeared to represent one of the selfreplicating PrP structures, our numerous attempts to amplify atypical PrPres in PMCAb failed (data not shown). We hypothesized that the $\mathrm{PrP}^{\mathrm{C}}$ glycoform ratio in $\mathrm{NBH}$ favors $\mathrm{PrP}^{\mathrm{Sc}}$ amplification but is disadvantageous for atypical PrPres, which is predominantly monoglycosylated. Indeed, when NBH was pretreated with PNGase F (Fig. 7A), atypical PrPres could be readily amplified (Fig. $7 B, C$ ). Pretreatment of NBH with PNGase F did not fully remove glycosyls but shifted the ratio of $\operatorname{PrP}^{\mathrm{C}}$ glycoforms from predominantly diglycosylated to predominantly monoglycosylated (Fig. 7A). The PMCAb format that uses PNGase F-pretreated NBH as a substrate is designated as dgPMCAb. Notably, much lower amounts of $\mathrm{PrP}^{\mathrm{Sc}}$ was amplified in dgPMCAb than in standard PMCAb seeded with the same brain material (Fig. $7 B)$. Nevertheless, the amounts of dgPMCAb-produced $\mathrm{PrP}^{\mathrm{Sc}}$ correlated well with the amounts of $\operatorname{PrP}^{\mathrm{Sc}}$ seeds in brain material: the 

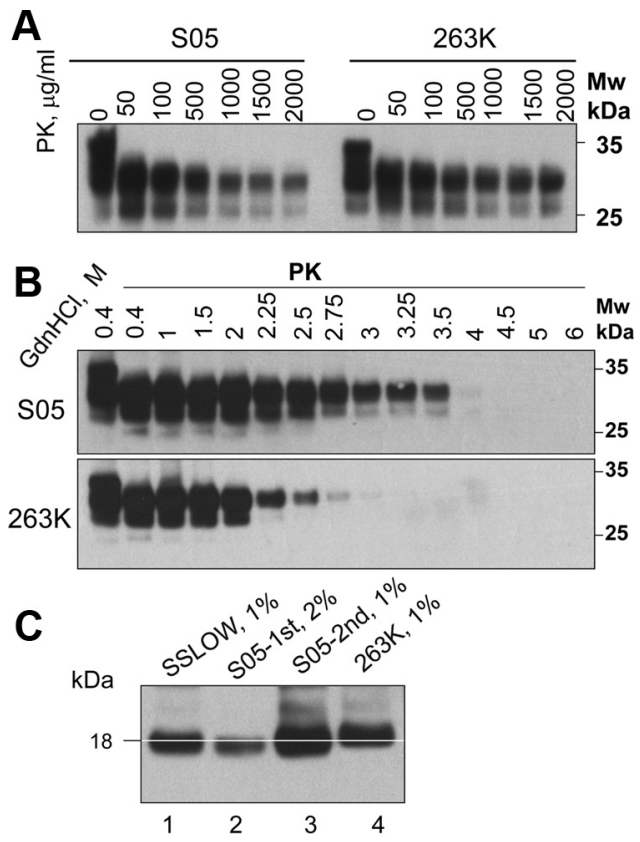

Figure 5. Analysis of S05 PrP Sc biochemical features. A, Analysis of PK-resistance. BHs from S05or 263K-inoculated animals were treated with increasing concentrations of glycerol-free proteinase $\mathrm{K}$ (Sigma, catalog no. P6556) in the presence of $0.25 \%$ SDS for $1 \mathrm{~h}$ at $37^{\circ} \mathrm{C}$. BH from the second passage of $\mathrm{SO} 5$ was used. $\boldsymbol{B}$, Analysis of $\mathrm{PrP}^{\mathrm{Sc}}$ conformational stability. One percent $\mathrm{BH}$ s from animals inoculated with 505 or $263 \mathrm{~K}$ were incubated with increasing concentrations of $\mathrm{GdnHCl}$ from 0.4 to $6 \mathrm{M}$ for $1 \mathrm{~h}$, as indicated, then diluted out of $\mathrm{GdnHCl}$, equilibrated for $1 \mathrm{~h}$ at room temperature, and digested with $20 \mu \mathrm{g} / \mathrm{ml} \mathrm{PK}$. Undigested brain material exposed to $0.4 \mathrm{M} \mathrm{GdnHCl}$ is provided as a reference. BH from the second passage of 505 was used. $C$, Analysis of a size of PrP Sc PK-resistant core. Two or one percent BHs from the first (lane2) or second (lane3) passages of S05, respectively, treated with PK and PNGase F and analyzed by Western blot. BHs from the SSLOW-inoculated (lane 1) or 263K-inoculated animals (lane 4) are shown as references. White line indicates the center of 505 unglycosylated PrP Sc. Western blots were stained with $3 \mathrm{~F} 4$ in all experiments.

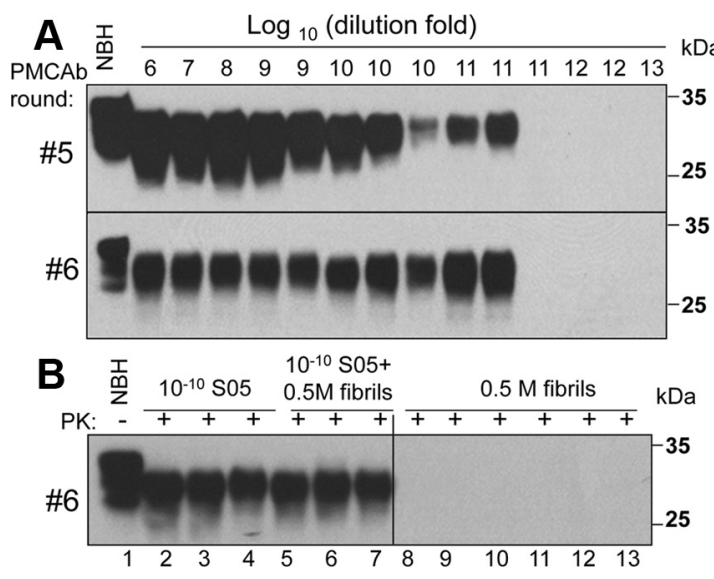

Figure 6. Preparations of $0.5 \mathrm{~m}$ fibrils have no detectible $\operatorname{PrP}^{{ }^{S c}} \cdot \boldsymbol{A}, S 05$ brain material was serially diluted to up to $10^{13}$-fold, and each dilution was subjected to five (top) or six rounds (bottom) of sPMCAb. Representative results are shown on Western blot stained with 3F4 antibody. B, sPMCAb reactions were seeded with $0.5 \mathrm{MrPrP}$ fibrils (lanes $8-13), 10^{10}$-fold diluted 505 brain material (lanes 2-4), or $0.5 \mathrm{~m}$ fibrils and $10^{10}$-fold diluted 505 brain material (lanes $5-7$ ); then six rounds of sPMCAb were conducted for each condition. The final concentration of $0.5 \mathrm{~m}$ fibrils in sPMCAb reaction was 2.5 $\mu \mathrm{g} / \mathrm{ml}$. Brains from clinically ill animals from $\mathrm{S} 05$ second passage were used for all experiments. Undigested $10 \% \mathrm{NBH}$ is provided as a reference. Western blots were stained with $3 \mathrm{~F} 4$.

reactions seeded with material \#3 showed the most abundant $\operatorname{PrP}{ }^{\mathrm{Sc}}$ signal, whereas the reactions seeded with material \#4 produced the least abundant signal (Figs. 2A, 7B). Again, no signs of atypical PrPres were detected in PMCAb seeded with the same brain material containing atypical PrPres (Fig. $7 B, C$ ). These results suggest that (1) atypical PrPres and $\mathrm{PrP}^{\mathrm{Sc}}$ are two self-replicating states, which compete for a substrate in dgPMCAb, and (2) dgPMCAb favors atypical PrPres, whereas standard $\mathrm{PrP}^{\mathrm{Sc}}$ had a selective advantage over atypical PrPres in standard PMCAb (Fig. $7 B, C$ ).

While both brain-derived PrPres and dgPMCAb-derived atypical PrPres were predominantly monoglycosylated, dgPMCAbderived PrPres displayed two PK-resistant unglycosylated bands instead of the one in brain-derived PrPres (Fig. $7 B, D$ ). These two bands were produced as a result of PK cleavage at two closely located sites. When dgPMCAb-derived PrPres was treated with higher concentration of PK, only a single unglycosylated band similar in size to unglycosylated brain-derived PrPres was observed (Fig. 7C). In fact, $0.5 \mathrm{M}$ fibrils, too, displayed two PK-digestion sites at a low PK concentration and a single site at high PK concentration (Fig. 2D).

\section{Fibrils $(0.5 \mathrm{M})$ triggered atypical PrPres in dgPMCAb}

To test whether $0.5 \mathrm{M}$ fibrils can trigger atypical PrPres in vitro, serial dgPMCAb was seeded with fibrils. Remarkably, the reactions seeded with $0.5 \mathrm{~m}$ fibrils produced the same PK-resistant band pattern as those observed in dgPMCAb seeded with brain material containing atypical PrPres (Fig. $7 B, E$ ). We performed numerous dgPMCA reactions using several independent preparations of $0.5 \mathrm{M}$ fibrils and several preparations of PNGase F-treated NBH substrate. In all experiments, $0.5 \mathrm{M}$ fibril-induced formation of atypical PrPres was robust. The PK-resistant band patterns produced by $0.5 \mathrm{~m}$ fibrils and brain-derived atypical PrPres in dgPMCAb were always remarkably similar if not identical. No PrPres bands were observed in nonseeded serial dgPMCAb reactions or serial dgPMCAb reactions seeded with hamster $\mathrm{rPrP}$ S-fibrils or fibrils produced from full-length mouse $\mathrm{rPrP}$ (Fig. $7 B$ ). These results illustrated that the process of inducing atypical PrPres in dgPMCAb was species specific and $0.5 \mathrm{M}$ fibril structure specific. Most important, these results supported the hypothesis that $0.5 \mathrm{M}$ fibrils gave rise to atypical PrPres in animals and that $0.5 \mathrm{M}$ fibrils and atypical PrPres are structurally very similar.

The attempts to use $0.5 \mathrm{M}$ fibril-induced atypical PrPres to seed $\mathrm{PrP}^{\mathrm{Sc}}$ in standard sPMCAb failed, pointing out that authentic $\mathrm{PrP}^{\mathrm{Sc}}$ was still absent in dgPMCA products of $\mathrm{rPrP}$ fibrils, nor was it generated in $\mathrm{SPMCAb}$. The fact that $\operatorname{PrP}^{\mathrm{Sc}}$ was observed in dgPMCAb products of amplification of brain-derived atypical PrPres (Fig. $7 B$ ) can be explained by the fact that $\operatorname{PrP}^{\mathrm{Sc}}$ was already present in those brains in small amounts (Fig. 2A).

\section{Amplification of atypical PrPres is RNA independent,} whereas amplification of $\operatorname{PrP}^{\mathrm{Sc}}$ is RNA dependent Successful amplification of atypical PrPres in gdPMCA seeded with $0.5 \mathrm{M}$ fibrils pointed to the fact that atypical PrPres was the first product of $\mathrm{PrP}^{\mathrm{C}}$ misfolding triggered by fibrils. Formation of atypical PrPres accomplishes an important step in triggering pathogenic process: switching from $r \operatorname{PrP}$ to $\operatorname{PrP}^{\mathrm{C}}$, a GPIanchored protein with complex glycosylation. The failure to produce $\operatorname{PrP}^{\mathrm{Sc}}$ in PMCAb upon seeding with dgPMCAb-derived atypical PrPres supports the idea that atypical PrPres and $\operatorname{PrP} \mathrm{P}^{\mathrm{Sc}}$ are structurally different.

Structural differences between atypical $\operatorname{PrPres}$ and $\operatorname{Pr} \mathrm{P}^{\mathrm{Sc}}$ could be highlighted by the differences in RNA dependency of their amplification. Previous studies established that amplification of hamster-adapted scrapie strains in PMCA was RNA dependent (Deleault et al., 2003, 2007, 2010). Two experimental formats were used here to test the effect of RNA. In the first format, serially diluted S05 materials containing atypical PrPres and $\operatorname{PrP} \mathrm{P}^{\mathrm{Sc}}$ were used to seed PMCAb or dgPMCAb reactions conducted in 
$\mathrm{NBH}$ or RNA-depleted NBH. In PMCAb (that selectively amplifies $\operatorname{PrP}^{\mathrm{Sc}}$ ), the amplification of S05 $\mathrm{PrP}^{\mathrm{Sc}}$ was observed only in $\mathrm{NBH}$ but not in RNA-depleted NBH (Fig. $8 A)$. The signal detected in RNA-depleted $\mathrm{NBH}$ was simply due to seed dilution (Fig. $8 A$ ). In dgPMCAb, amplification of atypical PrPres was observed in both $\mathrm{NBH}$ and RNA-depleted NBH, whereas robust amplification of $\mathrm{PrP}^{\mathrm{Sc}}$ was seen only in $\mathrm{NBH}$ (Fig. $8 B)$. RNA depletion substantially reduced the efficiency of $\mathrm{PrP}^{\mathrm{Sc}}$ amplification.

In an alternative format, dgPMCAb reactions were seeded with S05 materials containing atypical PrPres and $\mathrm{PrP}^{\mathrm{Sc}}$, and then four serial rounds were performed in NBH or RNA-depleted NBH (Fig. 8C). While atypical PrPres was steadily amplified in both conditions, $\operatorname{PrP}^{\mathrm{Sc}}$ could be only amplified in NBH containing RNA (Fig. 8C). Remarkably, just like brainderived atypical PrPres, $0.5 \mathrm{M}$ fibrilderived atypical PrPres could be also amplified equally well in the presence or absence of RNA (Fig. 8D). In summary, $\mathrm{rPrP}$ fibrils that were produced in the absence of RNA triggered in dgPMCA formation of PrPres, which was also able to amplify in the absence of RNA. Brainderived atypical PrPres was RNA independent as well, while S05 $\mathrm{PrP}^{\mathrm{Sc}}$ was RNA dependent, a feature typical for hamster prion strains.

\section{Discussion}

The current studies demonstrated that transmissible prion disease could be induced by rPrP fibrils with a structure different from that of $\operatorname{PrP}^{\mathrm{Sc}}$. A long, clinically silent stage accompanied by accumulation of atypical PrPres preceded development of clinical disease. Several lines of evidence suggested that the molecular mechanism leading to transmissible prion disease was fundamentally different from the previously known mechanisms, including the template-assisted conversion initiated by $\mathrm{PrP}^{\mathrm{Sc}}$ or the spontaneous conversion of $\mathrm{PrP}^{\mathrm{C}}$ into $\mathrm{PrP}^{\mathrm{Sc}}$. According to the template-assisted conversion mechanism (Cohen and Prusiner, 1998), the folding pattern of the newly produced $\mathrm{PrP} \mathrm{P}^{\mathrm{Sc}}$ accurately reproduces that of the $\mathrm{PrP}^{\mathrm{Sc}}$ template or seeds. In contrast, in the current studies, prion infection and transmissible disease emerged upon the inoculation of $0.5 \mathrm{M}$ fibrils with a structure different from that of $\operatorname{PrP}^{\mathrm{Sc}}$ (Spassov et al., 2006; Wille et al., 2009; Ostapchenko et al., 2010; Piro et al., 2011).

In previous studies, cellular cofactors including RNA and lipids were required for generating authentic $\operatorname{PrP}^{\mathrm{Sc}}$ structures in vitro from $\operatorname{PrP}^{\mathrm{C}}$ or $\mathrm{rPrP}$ (Deleault et al., 2007; Wang et al., 2010). Furthermore, cellular cofactors including RNAs are believed to be involved in $\operatorname{PrP}^{\mathrm{Sc}}$ replication (Deleault et al., 2003, 2005). In the current work, $\mathrm{rPrP}$ fibrils were produced in the absence of any cofactors. As judged from x-ray and FTIR analyses, the PrP folding pattern within $\mathrm{rPrP}$ fibrils is significantly different from that of $\operatorname{PrP}^{\text {Sc }}$ (Spassov et al., 2006; Wille et al., 2009; Ostapchenko et al., 2010). Moreover, application of ultra-sensitive PMCAb, which is capable of detecting single $\operatorname{PrP}^{\mathrm{Sc}}$ particles, demonstrated that preparations of $0.5 \mathrm{M}$ rPrP fibrils had no detectible $\operatorname{PrP}^{\mathrm{Sc}}$. $\mathrm{PMCAb}$ has been very useful for amplification of minuscule amounts of $\operatorname{PrP}^{\mathrm{Sc}}$, but when seeded with recombinant fibrils, no product was generated, suggesting that authentic $\operatorname{PrP}^{\mathrm{Sc}}$ was absent in the preparations of fibrils. On the other hand, $0.5 \mathrm{M}$ fibrils produced atypical PrPres in dgPMCA, proving their capacity to seed misfolding of $\mathrm{PrP}^{\mathrm{C}}$ and demonstrating their structural difference with authentic $\operatorname{PrP}^{\mathrm{Sc}}$.

When triggered by structures different from that of $\operatorname{Pr} \mathrm{P}^{\mathrm{Sc}}$, genesis of authentic $\mathrm{PrP}^{\mathrm{Sc}}$ appears to involve at least two major steps (Fig. 3D). The first step consisted of rPrP fibril-seeded formation of atypical PrPres. Similarities in size and position of the PK-resistant core suggest that atypical PrPres originated from that of rPrP fibrils (Fig. 2D). Moreover, $0.5 \mathrm{~m}$ fibrils triggered atypical PrPres in dgPMCAb with a PK resistance profile identical to that of brain-derived atypical PrPres. In opposite to $\operatorname{PrP}^{\mathrm{Sc}}$, amplification of both brain-derived and fibril-triggered atypical PrPres was RNA-independent.

The second step, the appearance of $\mathrm{PrP}^{\mathrm{Sc}}$, can be a result of a series of stochastic mutations of atypical PrPres structure and selection of the most favorable conformers that fit well to a par- 


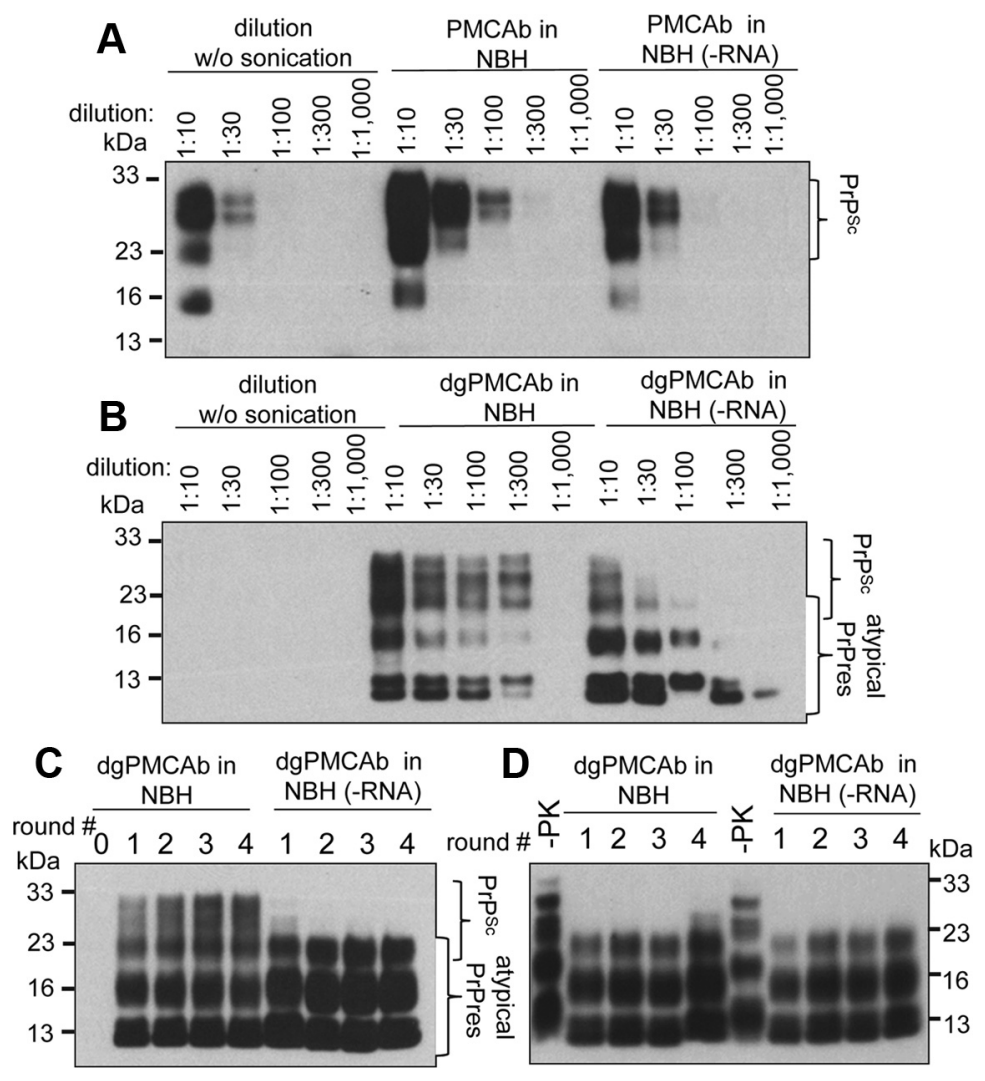

Figure 8. Analysis of RNA dependency of $\operatorname{PrP}^{\mathrm{Sc}}$ and atypical PrPres amplification. $\boldsymbol{A}, \boldsymbol{B}$, Analysis of amplification rate of $S 05$ $\operatorname{PrP}^{\mathrm{SC}}(\boldsymbol{A})$ or atypical PrPres $(\boldsymbol{B})$ in NBH or RNA-depleted NBH [NBH(-RNA)]. $\boldsymbol{A}$, S05 10\% brain material from animal \#3 was diluted 10-, 30-, 100-, 300-, or 1000-fold as indicated into 10\% NBH or RNA-depleted NBH and amplified for one PMCAb round. Dilution of seeds without amplification is shown as control. $\boldsymbol{B}$, Brain material from animal \#3 was first amplified in dgPMCAb for four serial rounds, and then dgPMCAb products were diluted 10-,30-, 100-, 300-, or 1000-fold, as indicated, into 10\% NBH or RNA-depleted NBH and amplified for one dgPMCAb round. Dilution of seeds without amplification is shown as control. C, Serial dgPMCAb reactions seeded with S05-derived atypical PrPres and conducted in NBH or RNA-depleted NBH. To prepare S05-derived atypical PrPres, serial dgPMCAb was seeded with $10^{3}$-fold diluted brain materials from animal \#3 and subjected to four rounds. Small amounts of $\operatorname{PrP}^{\mathrm{Sc}}$ could not be amplified after RNA depletion, whereas atypical PrPres amplifies well in both conditions. $D$, Serial dgPMCAb reactions seeded with $0.5 \mathrm{~m}$ fibril-derived atypical PrPres and conducted in NBH or RNA-depleted NBH. To prepare $0.5 \mathrm{M}$ fibril-derived atypical PrPres, serial dgPMCAb was seeded with $0.5 \mathrm{~m}$ fibrils and subjected to four rounds as shown in Figure $7 E$. The $0.5 \mathrm{~m}$ fibril-derived atypical PrPres amplifies equally well in both NBH and RNA-depleted NBH. In all experiments the reaction products were treated with $50 \mu \mathrm{g} / \mathrm{ml}$ PK. All Western blots were stained with SAF-84.

ticular cellular environment. The transition from atypical PrPres to $\mathrm{PrP}^{\mathrm{Sc}}$ is quite complicated and poorly understood. While achievable in the brain of animals over their life span, this transition may be very hard to simulate in vitro. While $\operatorname{PrP}^{\mathrm{C}}$ in hamster brain is predominantly diglycosylated, the distribution of $\mathrm{PrP}^{\mathrm{C}}$ glycoforms and their ratio vary in different brain regions and neuronal compartments as well as during neuronal differentiation (DeArmond et al., 1999; Russelakis-Carneiro et al., 2002; Monnet et al., 2003). dgPMCAb/PMCAb might not represent the environment at cellular sites where the transition from atypical PrPres to $\operatorname{PrP}^{\mathrm{Sc}}$ occurs. Indeed, $\operatorname{PrP}^{\mathrm{Sc}}$ was not generated in serial PMCAb seeded with $0.5 \mathrm{M}$ fibrils or with atypical PrPres triggered by fibrils in dgPMCAb. These results strongly supported earlier conclusions that authentic $\operatorname{PrP}^{\mathrm{Sc}}$ was absent in preparations of fibrils or in dgPMCAb products of fibrils. If the above mechanism is correct, $\mathrm{PrP}^{\mathrm{Sc}}$ can be considered as a relatively rare byproduct of replication of atypical PrPres. Variations in the amounts of $\mathrm{PrP}^{\mathrm{Sc}}$ within and between animal groups support the hypothesis of the stochastic nature of the second step. According to the proposed mechanism, after the first $\mathrm{PrP}^{\mathrm{Sc}}$ particles were formed, $\mathrm{PrP}^{\mathrm{Sc}}$ can replicate independently of atypical PrPres and eventu- ally outcompete its ancestor (Fig. 3D). An alternative hypothesis is that atypical PrPres represents a low stability, so-called class III strain that can readily mutate into a high stability strain upon serial passage. (Bruce and Dickinson, 1987). However, considering that the properties of atypical PrPres are so strikingly different from those of low or high stability class $\operatorname{PrP}^{\mathrm{Sc}}$, this possibility is unlikely.

Formation of $\mathrm{PrP}^{\mathrm{Sc}}$ in seeding events initiated by atypical PrPres is best described by a mechanism designated as "deformed templating" (Makarava et al., 2011). According to deformed templating, daughter fibrils or particles can acquire a folding pattern different from that of seeds (Makarava et al., 2009). The observations that atypical PrPres and $\mathrm{PrP}^{\mathrm{Sc}}$ preferred different $\mathrm{PrP}^{\mathrm{C}}$ glycoforms as a substrate and that atypical PrPres was RNA independent while $\mathrm{PrP}^{\mathrm{Sc}}$ was RNA dependent support the notion of significant structural differences between these forms. Substantial variation in amounts of $\mathrm{PrP}^{\mathrm{Sc}}$ at the end of the first passage suggests that the deformed templating events are relatively rare and stochastic.

The atypical PrPres described here was very similar to the atypical PrPres found in patients with sporadic CreutzfeldtJakob disease (Zou et al., 2003), atypical bovine spongiform encephalopathy $(\mathrm{H}$ BSE), which is believed to be sporadic in origin (Biacabe et al., 2007), or ovine scrapie (Baron et al., 2008). This current study suggests that atypical PrPres can replicate in animal brains and that its replication does not require $\mathrm{PrP}^{\mathrm{Sc}}$ assistance; therefore, it represents one of the transmissible PrP states. In current and previous studies on synthetic prions (Makarava et al., 2011), atypical PrPres always preceded $\operatorname{PrP}^{\mathrm{Sc}}$. No $\operatorname{PrP}^{\mathrm{Sc}}$ was found in atypical PrPres-negative animals. While accumulation of atypical PrPres alone was not pathogenic, its replication seems to represent a silent stage in the genesis of authentic $\operatorname{PrP}^{\mathrm{Sc}}$. Bearing in mind that much of the public health risk derives from long silent or asymptomatic stages (Peden et al., 2004; Comoy et al., 2008), detection of atypical PrPres should not be underestimated in developing prion detection strategies. This work introduces the first approach for selective amplification of atypical PrPres in vitro - dgPMCAb in RNA-depleted NBH. dgPMCAb should be a useful technique for establishing the relationship between atypical PrPres and $\mathrm{PrP}^{\mathrm{Sc}}$ in natural TSEs.

The hypothesis that amyloid structures significantly different from that of $\mathrm{PrP}^{\mathrm{Sc}}$ can trigger transmissible prion diseases has numerous clinical and epidemiological implications for understanding the origin of TSEs, including TSEs that are considered to be sporadic. The questions of great interest are whether all $\operatorname{PrP}$ amyloid structures are equally active in triggering $\operatorname{PrP}^{\mathrm{Sc}}$ and, if not, what is the spectrum of non- $\mathrm{PrP}^{\mathrm{Sc}}$ structures capable of inducing transmissible diseases in wild-type hosts? In contrast to $0.5 \mathrm{M}$ fibrils, inoculations of $2 \mathrm{M}$ fibrils or $\mathrm{S}$ fibrils did not lead to 
prion infection. Lack of any PrPres material including atypical PrPres in these two groups suggest these two structures were not effective in recruiting and/or converting $\operatorname{PrP}^{\mathrm{C}}$. Bearing in mind that all three amyloid states were formed within the same amino acid sequence, the differences in their pathogenic activity should be attributed to their individual fibril-specific physical features.

Previous studies on synthetic prions that employed transgenic mice established a correlation between conformational stability of rPrP fibrils and the incubation time to disease (Colby et al., 2009, 2010). Fibrils with low conformational stability were found to cause the disease within a shorter incubation time when compared to the high stability fibrils (Colby et al., 2009). In addition, strain-specific conformational stability of $\mathrm{PrP}^{\mathrm{Sc}}$ was proposed as one of the physical features that control prion amplification rate and incubation time to disease (Legname et al., 2005; Makarava et al., 2010; Ayers et al., 2011; Gonzalez-Montalban et al., 2011b). The current finding that $2 \mathrm{M}$ or $\mathrm{S}$ fibrils with a high stability failed to trigger prion infection strongly support the previously established correlation. As evident from FTIR and x-ray diffraction analyses, the PrP folding pattern within S fibrils closely resembled that of $\operatorname{PrP}^{\mathrm{Sc}}$ (Ostapchenko et al., 2010; Wille et al., 2009). Unexpectedly, $S$ fibrils failed to trigger prion infection. $S$ fibrils also failed to trigger atypical PrPres in vitro. These data suggest that conformational stability of $\mathrm{rPrP}$ fibrils appears to be more important for triggering pathogenic process than an apparent structural similarity between inoculated material and $\mathrm{PrP}^{\mathrm{Sc}}$. Conformational stability appears to be linked to the fibril's mechanical properties, such as its intrinsic fragility (Baskakov and Breydo, 2007; Sun et al., 2008). One might speculate that $2 \mathrm{M}$ or S fibrils failed to recruit $\mathrm{PrP}^{\mathrm{C}}$ because of their low fragmentation rate.

The current studies illustrate that transmissible prion disease can emerge according to a previously unknown mechanism that is different from the spontaneous conversion of $\operatorname{PrP}^{\mathrm{C}}$ to $\mathrm{PrP}^{\mathrm{Sc}}$ or the template-assisted conversion initiated by authentic $\operatorname{PrP}^{\mathrm{Sc}}$. The key features of the new mechanism are: (1) the pathogenic process is initiated by amyloid structures different from $\operatorname{PrP}^{\mathrm{Sc}}$; (2) it is accompanied by a long clinically silent stage; and (3) it is characterized by the accumulation of atypical transmissible PrP states that display limited neurotoxicity before $\operatorname{PrP}^{\mathrm{Sc}}$ emerges. The current work also shows that prion infection can be induced in wild-type animals by $\mathrm{rPrP}$ fibrils produced in vitro in the absence of any cellular cofactors or $\mathrm{PrP}^{\mathrm{Sc}}$ seeds.

\section{References}

Aguzzi A, Rajendran L (2009) The transcellular spread of cytosolic amyloids, prions, and prionoids. Neuron 64:783-790.

Ayers JI, Schutt CR, Shikiya RA, Aguzzi A, Kincaid AE, Bartz JC (2011) The strain-encoded relationship between PrP replication, stability and processing in neurons is predictive of the incubation period of disease. PLoS Pathog 7:e1001317.

Baron T, Bencsik A, Vulin J, Biacabe AG, Morignat E, Verchere J, Betemps D (2008) A C-terminal protease-resistant prion fragment distinguishes ovine "CH1641-like" scrapie from bovine classical and L-type BSE in ovine transgenic mice. PLoS Pathog 4:e1000137.

Baskakov IV, Breydo L (2007) Converting the prion protein: What makes the protein infectious. Biochim Biophys Acta 1772:692-703.

Biacabe AG, Jacobs JG, Bencsik A, Langeveld JP, Baron TG (2007) H-type bovine spongiform encephalopathy: complex molecular features and similarities with human prion diseases. Prion 1:61-68.

Bocharova OV, Breydo L, Parfenov AS, Salnikov VV, Baskakov IV (2005a) In vitro conversion of full length mammalian prion protein produces amyloid form with physical property of PrPSc. J Mol Biol 346:645-659.

Bocharova OV, Breydo L, Salnikov VV, Gill AC, Baskakov IV (2005b) Synthetic prions generated in vitro are similar to a newly identified subpopulation of PrPSc from sporadic Creutzfeldt-Jakob disease $\operatorname{PrP}^{\mathrm{Sc}}$. Protein Sci 14:1222-1232.
Bruce ME, Dickinson AG (1987) Biological evidence that the scrapie agent has an independent genome. J Gen Virol 68:79-89.

Cohen FE, Prusiner SB (1998) Pathologic conformations of prion proteins. Annu Rev Biochem 67:793-819.

Colby DW, Giles K, Legname G, Wille H, Baskakov IV, DeArmond SJ, Prusiner SB (2009) Design and construction of diverse mammalian prion strains. Proc Acad Natl Sci U S A 106:20417-20422.

Colby DW, Wain R, Baskakov IV, Legname G, Palmer CG, Nguyen HO, Lemus A, Cohen FE, DeArmond SJ, Prusiner SB (2010) Proteasesensitive synthetic prions. PLoS Pathog 6:e1000736.

Comoy EE, Casalone C, Lescoutra-Etchegaray N, Zanusso G, Freire S, Marcé D, Auvré F, Ruchoux MM, Ferrari S, Monaco S, Salès N, Caramelli M, Leboulch P, Brown P, Lasmézas CI, Deslys JP (2008) Atypical BSE (BASE) transmitted from asymptomatic aging cattle to a primate. PLoS One 3:e3017.

DeArmond SJ, Qiu Y, Sánchez H, Spilman PR, Ninchak-Casey A, Alonso D, Daggett V (1999) $\operatorname{PrP}^{\mathrm{C}}$ glycoform heterogeneity as a function of brain region: implications for selective targeting of neurons by prion strains. J Neuropathol Exp Neurol 58:1000-1009.

Deleault AM, Deleault NR, Harris BT, Rees JR, Supattapone S (2008) The effects of prion protein proteolysis and disaggregation on the strain properties of hamster scrapie. J Gen Virol 89:2642-2650.

Deleault NR, Lucassen RW, Supattapone S (2003) RNA molecules stimulate prion protein conversion. Nature 425:717-720.

Deleault NR, Geoghegan JC, Nishina K, Kascsak R, Williamson RA, Supattapone S (2005) Protease-resistant prion protein amplification reconstituted with partially purified substrates and synthetic polyanions. J Biol Chem 280:26873-26879.

Deleault NR, Harris BT, Rees JR, Supattapone S (2007) Formation of native prions from minimal components in vitro. Proc Acad Natl Sci U S A 104:9741-9746.

Deleault NR, Kascsak R, Geoghegan JC, Supattapone S (2010) Speciesdependent differences in cofactor utilization for formation of the protease-resistant prion protein in vitro. Biochemistry 49:3928-3934.

Frost B, Diamond MI (2010) Prion-like mechanisms on neurodegenerative diseases. Nat Rev Neurosci 11:155-159.

Gonzalez-Montalban N, Makarava N, Ostapchenko VG, Savtchenko R, Alexeeva I, Rohwer RG, Baskakov IV (2011a) Highly Efficient Protein Misfolding Cyclic Amplification. PLoS Pathogen 7:e1001277.

Gonzalez-Montalban N, Makarava N, Savtchenko R, Baskakov IV (2011b) Relationship between conformational stability and amplification efficiency of prions. Biochemistry 50:7933-7940.

Jackson WS, Borkowski AW, Faas H, Steele AD, King OD, Watson N, Jasanoff A, Lindquist S (2009) Spontaneous generation of prion infectivity in fatal familial insomnia knockin mice. Neuron 63:438-450.

Kong Q, Surewicz WK, Petersen RB, Zou W, Chen SG, Gambetti P, Parchi P Capellari S, Goldfarb L, Montagna P, Lugaresi E, Piccardo P, Ghetti B (2004) Inherited prion diseases. In: Prion biology and diseases (Prusiner SB, ed), pp 673-775. Cold Spring Harbor, NY: Cold Spring Harbor Laboratory.

Legname G, Nguyen H-OB, Baskakov IV, Cohen FE, DeArmond SJ, Prusiner SB (2005) Strain-specified characteristics of mouse synthetic prions. Proc Natl Acad Sci U S A 102:2168-2173.

Makarava N, Baskakov IV (2008) The same primary structure of the prion protein yields two distinct self-propagating states. J Biol Chem 283:15988-15996.

Makarava N, Ostapchenko VG, Savtchenko R, Baskakov IV (2009) Conformational switching within individual amyloid fibrils. J Biol Chem 284:14386-14395.

Makarava N, Kovacs GG, Bocharova O, Savtchenko R, Alexeeva I, Budka H, Rohwer RG, Baskakov IV (2010) Recombinant prion protein induces a new transmissible prion disease in wild type animals. Acta Neuropathol 119:177-187.

Makarava N, Kovacs GG, Savtchenko R, Alexeeva I, Budka H, Rohwer RG, Baskakov IV (2011) Genesis of mammalian prions: from non-infectious amyloid fibrils to a transmissible prion disease. PLoS Pathog 7:e1002419.

Makarava N, Savtchenko R, Alexeeva I, Rohwer RG, Baskakov IV (2012) Fast and ultrasensitive method for quantitating prion infectivity titer. Nat Commun 3:741.

Miller G (2009) Could they all be prion diseases? Science 326:1337-1339.

Monnet C, Marthiens V, Enslen H, Frobert Y, Sobel A, Mège RM (2003) 
Heterogeneity and regulation of cellular prion protein glycoforms in neuronal cell lines. Eur J Neurosci 18:542-548.

Ostapchenko VG, Sawaya MR, Makarava N, Savtchenko R, Nilsson KP, Eisenberg D, Baskakov IV (2010) Two amyloid states of the prion protein display significantly different folding patterns. J Mol Biol 400:908-921.

Peden AH, Head MW, Ritchie DL, Bell JE, Ironside JW (2004) Preclinical vCJD after blood transfusion in a PRNP codon 129 heterozygous patient. Lancet 364:527-529.

Piro JR, Wang F, Walsh DJ, Rees JR, Ma J, Supattapone S (2011) Seeding specificity and ultrastructural characteristics of infectious recombinant pPrions. Biochemistry 50:7111-7116.

Prusiner SB (1997) Prion diseases and the BSE crisis. Science 278:245-251.

Prusiner SB, Scott MR (1997) Genetics of prions. Annu Rev Genet 31:139-175.

Russelakis-Carneiro M, Saborio GP, Anderes L, Soto C (2002) Changes in the glycosylation pattern of prion protein in murine scrapie. J Biol Chem 277:36872-36877.

Saá P, Castilla J, Soto C (2006) Ultra-efficient replication of infectious prions by automated protein misfolding cyclic amplification. J Biol Chem 281:35245-35252.
Sigurdson CJ, Nilsson KP, Hornemann S, Heikenwalder M, Manco G, Schwartz P, Ott D, Rulicke T, Liberski T, Julius C, Falsiq J, Stitz L, Wuthrich K, Aguzzi A (2009) De novo generation of a transmissible spongiform encephalopathy by mouse transgenesis. Proc Acad Natl Sci U S A 106:304-309.

Spassov S, Beekes M, Naumann D (2006) Structural differences between TSEs strains investigated by FT-IR spectroscopy. Biochim Biophys Acta 1760:1138-1149.

Sun Y, Makarava N, Lee CI, Laksanalamai P, Robb FT, Baskakov IV (2008) Conformational stability of PrP amyloid firbils controls their smallest possible fragment size. J Mol Biol 376:1155-1167.

Wang F, Wang X, Yuan CG, Ma J (2010) Generating a Prion Bacterially Expressed Recombinant Prion Protein. Science 327:1132-1135.

Wille H, Bian W, McDonald M, Kendall A, Colby DW, Bloch L, Ollesch J, Borovinskiy AL, Cohen FE, Prusiner SB, Stubbs G (2009) Natural and synthetic prion structure from X-ray fiber diffraction. Proc Acad Natl Sci U S A 106:16990-16995.

Zou WQ, Capellari S, Parchi P, Sy MS, Gambetti P, Chen SG (2003) Identification of novel proteinase K-resistant C-terminal fragments of $\operatorname{PrP}$ in Creutzfeldt-Jakob disease. J Biol Chem 278:40429-40436. 